\title{
Surgical treatment of giant pituitary adenomas
}

\author{
L. S Y M O N, J . J A K U B O WS K I, A N D B. K E N D A L L \\ From the Gough Cooper Department of Neurological Surgery and the Lysholm Radiological \\ Department, The National Hospital, London
}

SUMM ARY Multidirectional extension and invasive spread are important features of giant pituitary adenomas. Operability cannot be established merely by determining the size of the most prominent part of the tumour. Detailed radiological evaluation with plain films, computed tomography, angiography, and air studies all contribute to evaluation of the precise anatomy before surgery. In the final decision risks of surgical treatment must be balanced against the patient's age and prospects of long-term useful survival. Unfavourable cases for surgical treatment in our hands were those tumours embedded in the hypothalamus with thalamic and posterior extensions. Partial removal of such cases gave poor results. Where the mass proves soft, radical excision may be possible, but not otherwise. Limited biopsy for histological study, followed by a shunt procedure and x-ray therapy seems still the only recourse.

The giant pituitary adenoma has long been recognised as a formidable surgical problem. Jefferson (1940) presented 12 personal cases and discussed the natural history of these tumours and the difficulties of surgical treatment. His view was that very large suprasellar extensions usually indicated malignancy, and that this was a factor influencing high surgical mortality $(35 \%)$. Bakay (1950) found that mortality in Olivecrona's series of pituitary tumours was also greatly influenced by the presence of large suprasellar extensions. A more recent series (Wirth et al., 1974) reported a mortality rate of $18 \%$ in "large tumours." Such tumours frequently spread through their capsule, may infiltrate basal structures, nasopharynx, or orbits (Jefferson, 1955), and occasionally give rise to remote secondary deposits within the central nervous system (Ogilvy and Jakubowski, 1973).

Jefferson's view expressed in 1969 was that surgery was contraindicated when the surface area of a suprasellar extension as shown on an unmagnified lateral projection pneumoencephalcgram exceeded seven square centimetres and when the summit of the tumour was within six millimetres of the foramen of Monro. At this time he introduction an index defined as the ratio between the area of the suprasellar extension on a midline tomogram and the distance between the foramen

Address for reprint requests: Professor Lindsay Symon, Department of Neurological Surgery, Institute cf Neurology, The National Hospital, Queen Square, London WC1 N 3BG.

Accepted 24 April 1979 of Monro and the tumour, postulating that when this index exceeded unity, surgery was contraindicated, and deep $x$-ray treatment was the method of choice. Results over the next nine years supported this view (Jefferson, 1978). Kunicki et al. (1975), however, believed that radical surgery should be attempted in all patients with a general medical condition giving good prospects of longterm useful survival, and broad general support to at least partial excision was also expressed by Guiot and Derome (1976).

\section{Patients and methods}

Sixteen, from a total series of 117 pituitary tumours surgically treated during the period from 1968 to 1978, were selected as giant lesions on the basis of (1) extension more than $40 \mathrm{~mm}$ from the midline of the jugum sphenoidale in any direction or within $6 \mathrm{~mm}$ of the foramina cf Monro on midline tomography, and (2) lobules of intracranial tumour extending suprasellarly in more than two directions.

All cases were treated by one team using the same surgical and anaesthetic techniques. The clinical analysis of the patients is shown in Table 1 . The age of the cases ranged from 22 to 65 years and only three were over 60 years of age.

The patients were referred for treatment because of visual deterioration with two exceptions. Case 3 presented with pituitary apoplexy caused by haemorrhagic infarction of the tumour; it is 


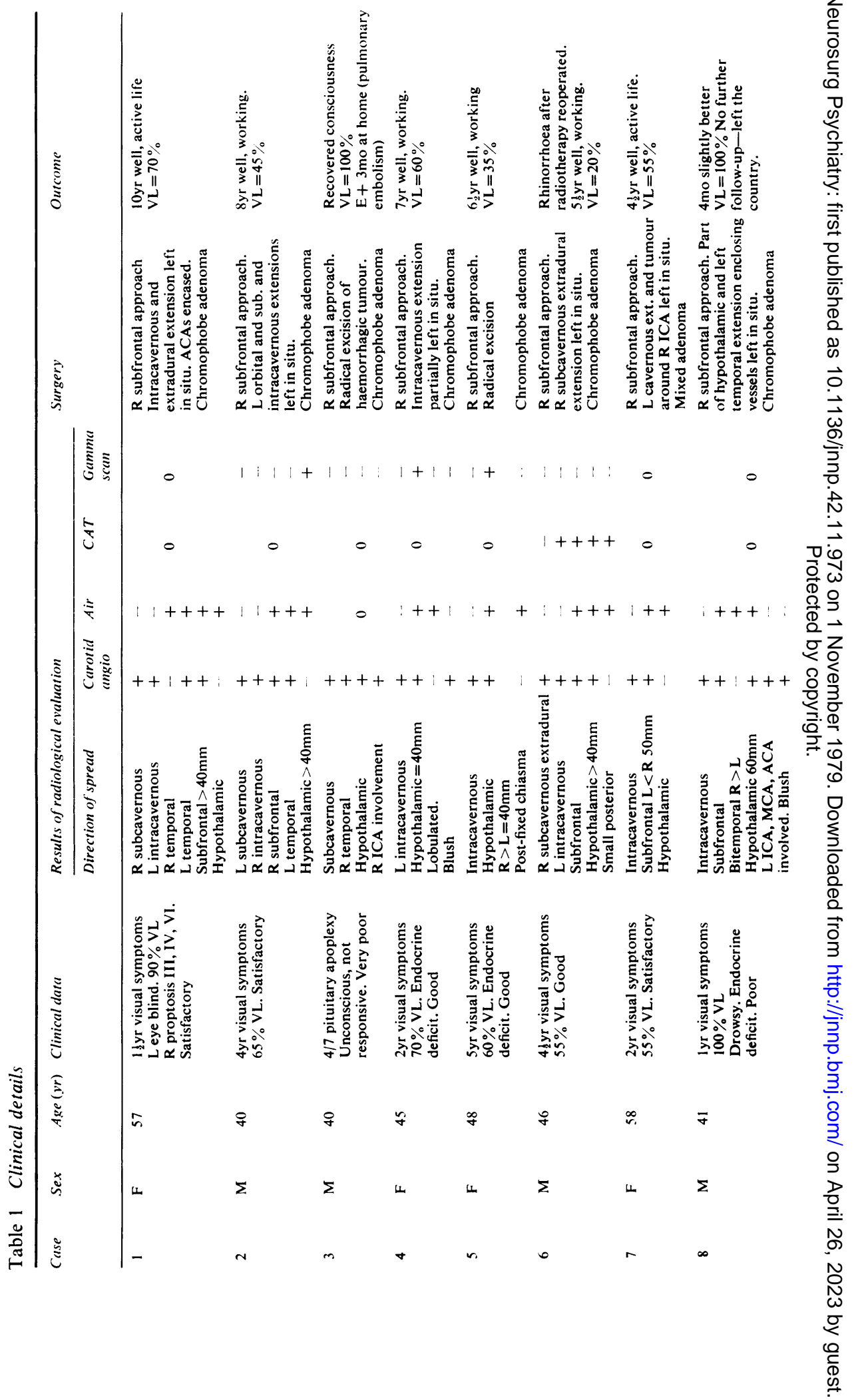



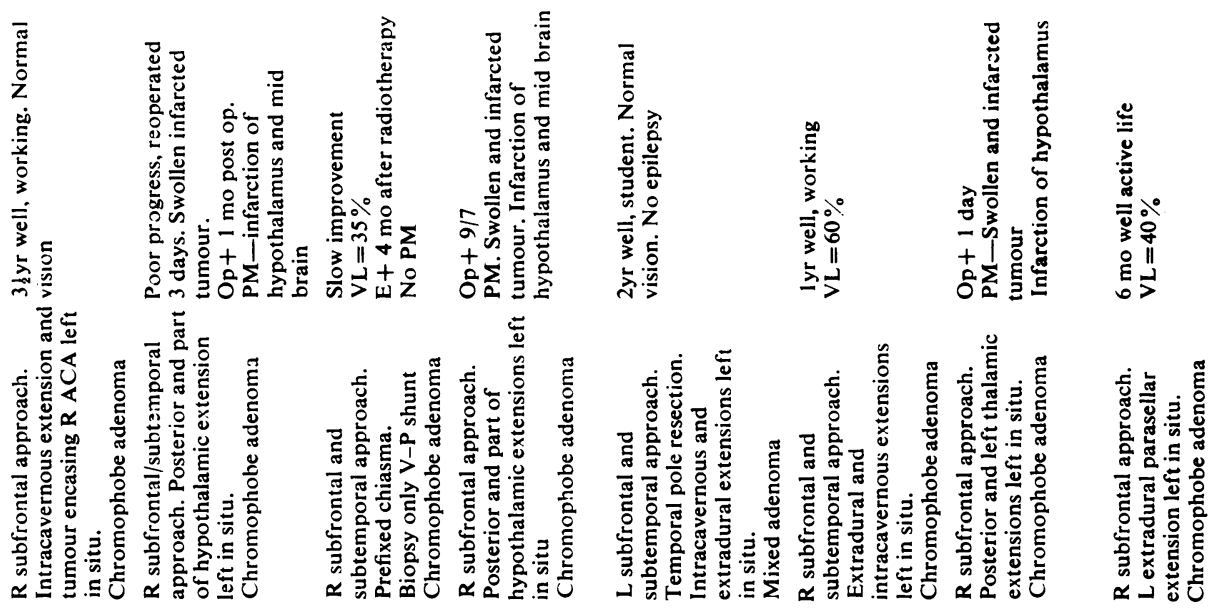

$+10$
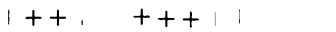

.....

$+++$

$1+t$
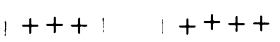

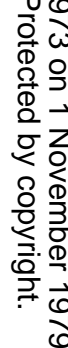
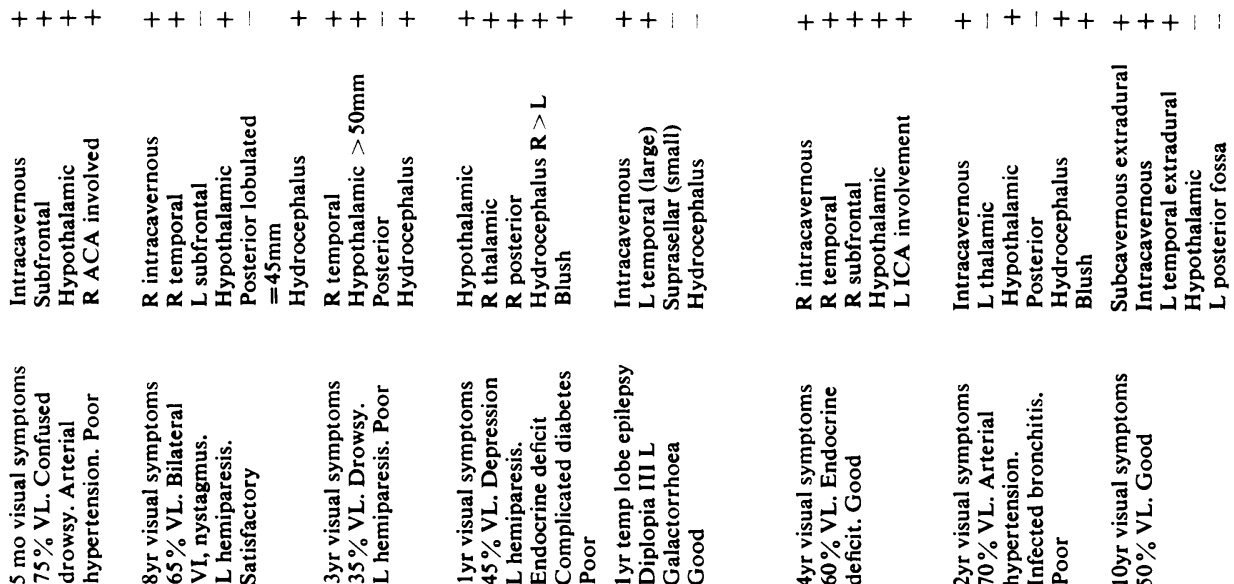

畜

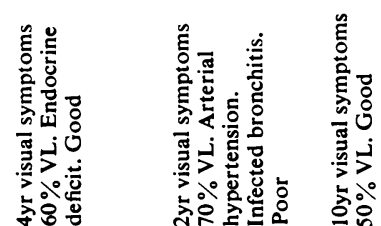

$$
\text { F }
$$

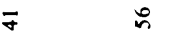

$\ddot{6}$

क

ส

$\checkmark$

3

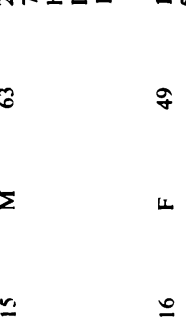

$\Sigma$

음

$=\quad \simeq$

$\underline{n}$

$\pm$ 
not known whether he had visual disturbance previously. Case 13 suffered only from temporal lobe epilepsy and galactorrhoea.

The length of history varied from four months to eight years (mean 2 $\frac{1}{2}$ years). The general medical condition of 10 cases was good or satisfactory; in the other six it was poor because of longstanding diminished level of consciousness, intercurrent infection, or the like. Five patients had clinical evidence of severe hypopituitarism, four had disturbed consciousness, and six patients had other neurological deficits involving weakness of limbs (three), nystagmus (one), proptosis (one), and weakness of ocular movement (three). The percentage of visual loss was scored using a points system (Symon and Jakubowski, 1979). One patient (case 13) had no visual deficit. Only two patients displayed a visual deficit less than $50 \%$; 12 had severe visual loss and one was completely blind.

Skull radiographs and angiography were performed in all cases and air study (ventriculography or encephalography) in 14 cases. Air study was thought contraindicated in the very ill patient with pituitary apoplexy, and was omitted in one patient in which angiography and computed tomography (CAT) gave adequate information for choice of treatment. Isotope scan was performed in eight and CAT scan in seven cases.

\section{ASSESSMENT OF THE EXTENT OF TUMOUR SPREAD}

Plain films These showed expansion and erosion of the sella turcica in all cases, of particular significance in differential diagnosis since further studies showing massive basal tumours or major anterior or lateral extension sometimes erroneously suggested the diagnosis of meningioma or chordoma. Eight patients had erosion of one or both lesser wings of the sphenoid sometimes associated with destruction of the orbital roof and ethmoid sinuses (Fig. 1) and, in two cases, the apex of a petrous bone. In three of these cases there was a large soft tissue mass in the sphenoidal sinuses and a similar mass occurred in three other cases. In two, tumour extended into the nasopharynx. Extensive erosion indicated the invasive nature of these tumours (Table 2).

$C A T$ scan The most accurate radiological assessment of the size, shape, and many of the relationships of these tumours was given by CAT after intravenous injection of contrast medium. Some lobules shown by CAT were not readily visible on pneumoencephalography or angiography. Predominantly unilateral or anterior extension with relatively minor suprasellar masses, however, could lead to a mistaken diagnosis of meningioma if the CAT appearances were considered in iso-尺 lation. In all these patients, however, enlargemento of the sella turcica and extension of the tumouro

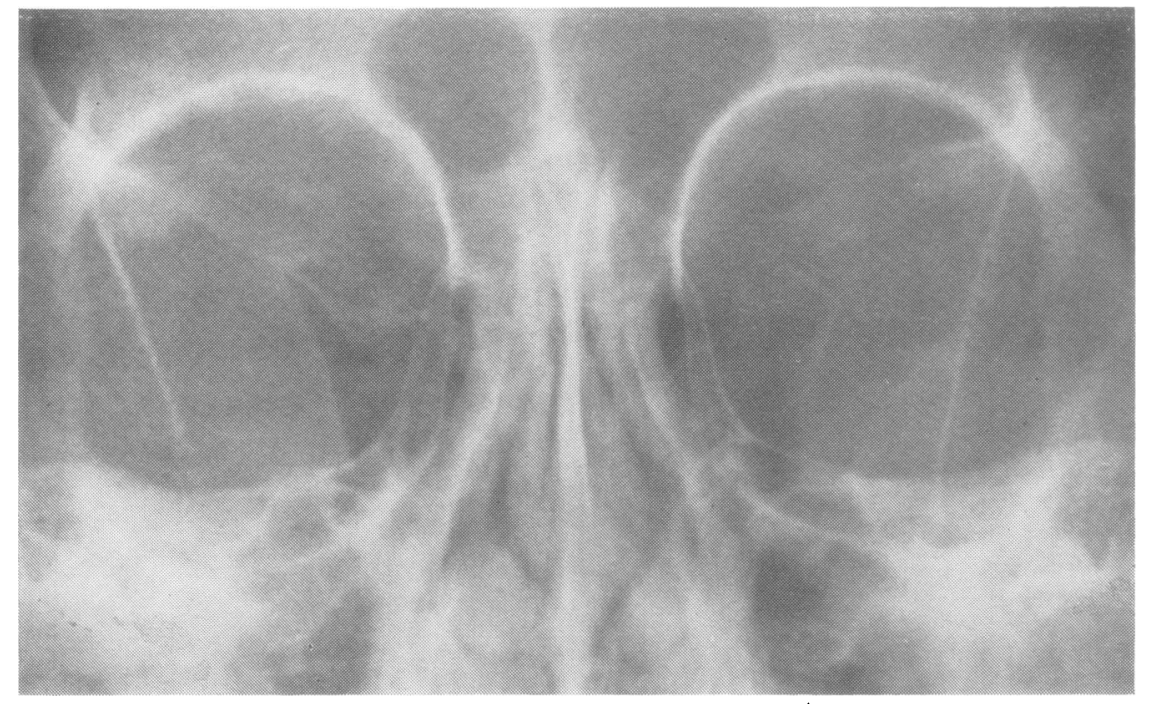

Fig. 1 Plain skull radiograph (case 2). There is marked erosion of the medial half of the left lesser wing of sphenoid and of the adjacent part of the roof of the orbit. The inferior border of the right lesser wing of sphenoid is eroded with widening of the superior orbital fissure. 
Table 2 Plain skull radiographic changes

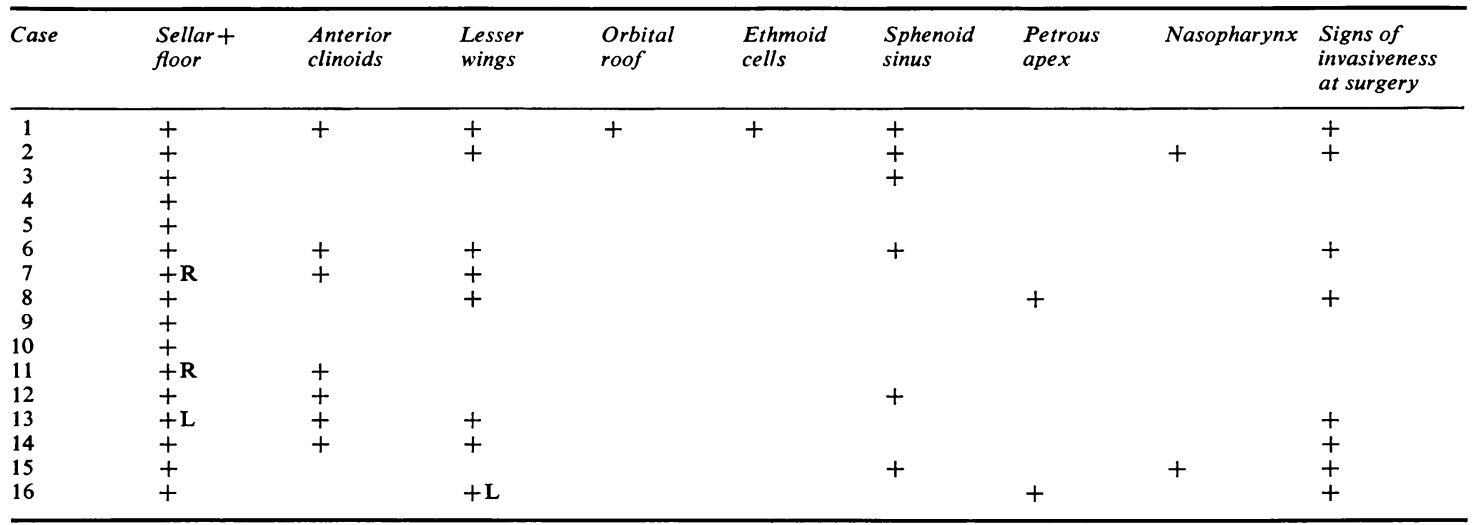

from it could be identified and this finding should always suggest the strong possibility of a pituitary adenoma. Review of the plain films in association with CAT scan clarifies the diagnosis.

On CAT the tumour was of homcgeneous slightly increased attenuation in four cases, and in one other it was of mixed high and isodense attenuation. One tumour was isodense and another of mixed iso- and low attenuation. After intravenous contrast medium (bolus injection of $60 \mathrm{ml}$ sodium iothalamate 420), all except one, in which a central region remained isodense, enhanced evenly. There was no relationship between the attenuation before and/or after intravenous con- trast medium, nor incidentally of isotope uptake, and the consistency and vascularity of the tumour found at surgery so that detailed observations of attenuation appear to have limited practical importance (Table 3 ).

Occasionally low attenuation non-enhancing regions may suggest cystic or necrotic changes, but such regions may be solid (Fig. 2) and cysts may be isodense. There was no brain oedema surrounding any of our pituitary adenomas. Such oedema occurs in some $20 \%$ of suprasellar meningiomas and may be a factor in differentia! diagnosis.

Angiography None of these particular patients

Table 3 Correlation of radiological and surgical findings

\begin{tabular}{|c|c|c|c|c|c|c|c|c|c|}
\hline \multirow[t]{2}{*}{ Case } & \multirow{2}{*}{$\begin{array}{l}\text { Gamma } \\
\text { scan }\end{array}$} & \multicolumn{2}{|c|}{$C A T$ scan } & \multicolumn{3}{|l|}{ At surgery } & \multirow{2}{*}{$\begin{array}{l}\text { Angiographic } \\
\text { vascularity } \\
\text { of tumour }\end{array}$} & \multicolumn{2}{|c|}{ Encasement of vessels } \\
\hline & & Plain & Enhanced & Consistency & Cysts & Vascularity & & Angiographic & At surgery \\
\hline 1 & 0 & 0 & 0 & Soft & - & - & - & $\begin{array}{l}\text { R ICA } \\
+\end{array}$ & $\begin{array}{l}\text { R ICA + ACAs } \\
+\end{array}$ \\
\hline 2 & + & 0 & 0 & Soft & - & - & - & $\begin{array}{l}\text { LICA } \\
+\end{array}$ & $\begin{array}{l}\text { LICA } \\
+\end{array}$ \\
\hline 3 & - & 0 & 0 & Soft & $+\mathrm{Ha}$ & ++ & - & $\begin{array}{l}\text { R ICA } \\
+\end{array}$ & $\begin{array}{l}\text { R ICA } \\
+\end{array}$ \\
\hline $\begin{array}{l}4 \\
5\end{array}$ & $\begin{array}{l}+ \\
+\end{array}$ & $\begin{array}{l}0 \\
0\end{array}$ & $\begin{array}{l}0 \\
0\end{array}$ & $\begin{array}{l}\text { Soft } \\
\text { Soft }\end{array}$ & $\begin{array}{l}- \\
-\end{array}$ & \pm+ & \pm & 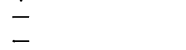 & $\overline{-}$ \\
\hline 6 & - & $\mathbf{H}$ & 0 & Hard & - & $\overline{-}$ & - & R ICA & $\bar{R}$ ICA \\
\hline 7 & 0 & 0 & 0 & $\begin{array}{l}\text { Hard } \\
\text { Rubbery }\end{array}$ & - & ++ & $\stackrel{+}{\text { Capsule }}$ & - & $\begin{array}{l}+ \\
+ \\
+\end{array}$ \\
\hline 8 & 0 & 0 & 0 & Hard & - & - & ++ & $\begin{array}{l}\text { ACAs, L ICA } \\
+\end{array}$ & $\begin{array}{l}\text { ACAs, ICA, MCA } \\
+\end{array}$ \\
\hline 9 & + & 0 & 0 & Sof $t$ & - & + & - & $\begin{array}{l}\text { ACAs } \\
+\end{array}$ & $\begin{array}{l}\text { ACAs } \\
+\end{array}$ \\
\hline 10 & 0 & 0 & 0 & Soft & - & ++ & - & - & - \\
\hline 11 & + & $\mathbf{I} / \mathbf{L}$ & 0 & Hard & - & + & - & - & - \\
\hline 12 & + & $\mathbf{H}$ & + & Hard & - & ++ & ++ & - & - \\
\hline 13 & 0 & $\mathbf{H}$ & + & Soft & - & + & - & $\begin{array}{l}\text { ACAs + choroid } \\
+\end{array}$ & $\begin{array}{l}\text { ACAs + choroid } \\
+\end{array}$ \\
\hline $\begin{array}{l}14 \\
15\end{array}$ & $\begin{array}{l}0 \\
0\end{array}$ & ${ }_{\mathbf{H} / \mathbf{I}}$ & + & Soft & - & ++ & - & - & - \\
\hline $\begin{array}{l}15 \\
16\end{array}$ & 0 & $\begin{array}{l}\mathrm{H} / \mathbf{1} \\
\mathrm{H}\end{array}$ & $\frac{ \pm}{t}$ & $\begin{array}{l}\text { Hard } \\
\text { Soft }\end{array}$ & - & \pm+ & \pm & $\overline{\mathrm{L}}_{\mathrm{ICA}}$ & $\overline{\mathrm{I}}$ \\
\hline & & & & & & & & + & + \\
\hline
\end{tabular}




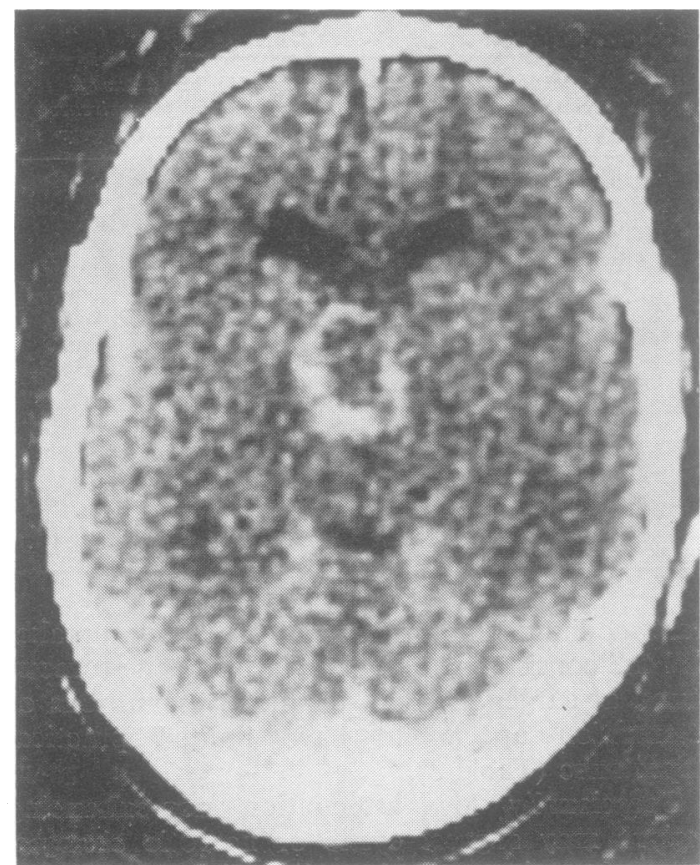

Fig. 2 Computed tomogram after intravenous contrast medium (case 15). There is a large mass in the suprasellar region. The periphery of the mass was of increased attenuation and enhanced after intravenous contrast medium. The centre of the mass is isodense with cerebral substance and does not enhance. There is no hydrocephalus.

had incidental aneurysms (Jakubowski and Kendall, 1978). Circumferential constriction of cerebral arteries adjacent to the tumour was shown on eight angiograms (Fig. 3). Extensive cuffing by tumour was confirmed at operation but it was also present around the anterior cerebral arteries in two other patients and around the middle cerebral artery in another patient in whom it was not evident on angiograms (Table 3 ). Narrowing or occlusion of major arteries in relation to tumour may be a factor in deciding against surgery. There was a poor relationship between the degree of vascularity shown on angiograms and the amount of bleeding or apparent vascularity of the tumour at surgery (Table 3). Magnification angiography, which shows the relation and size of vessels in the vicinity of the tumour, is helpful in the planning of surgical access (Fig. 4). Extension beneath or through the cavernous sinus, elevating the cavernous segment of the carotid artery and producing an extradural mass in the middle fossa, was identified on angiograms in five

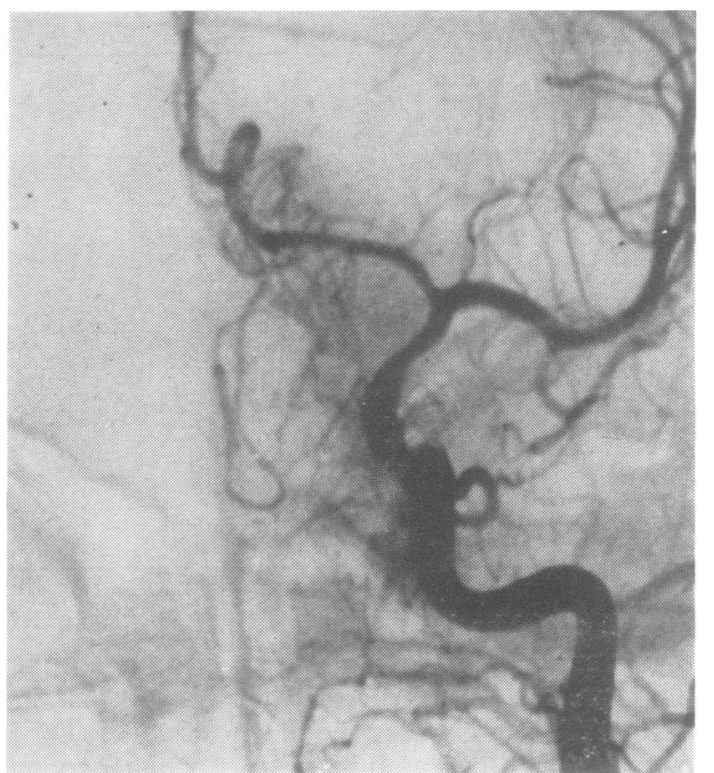

Fig. 3 Left carotid angiogram, arterial phase, antero-posterior projection (case 8). There is lateral displacement of the intracavernous and supraclinoid segments of the internal carotid artery and elevation of the first part of the anterior cerebral artery. There is tumour blush supplied by the inferior hypophyseal branch of the internal carotid artery. The tumour has extended around the supraclinoid carotid and the proximal part of the anterior cerebral artery causing narrowing.

cases. Such extensions were excluded from surgical consideration.

Air study With two exceptions, including one pneumoencephalogram in which the ventricular system did not fill, air studies showed the relation of the chiasm to the tumour. In case 11 the optic recess was displaced to the left and $15 \mathrm{~mm}$ above and behind the tuberculum sellae. At surgery the anterior edge of the chiasm was in fact too close to the sella turcica to allow access to the tumour from the frontal approach. The discrepancy was caused by an unusually broad chiasm.

The air study alone usually revealed the extent of tumour lobulation, but important subfrontal and hypothalamic extensions could be present without displacement of the third ventricle so that the air studies should be examined in conjunction with the CAT. Multidirectional tomography is important in the precise identification of the chiasmatic recess; perichiasmatic or especially retrochiasmatic extension with a 


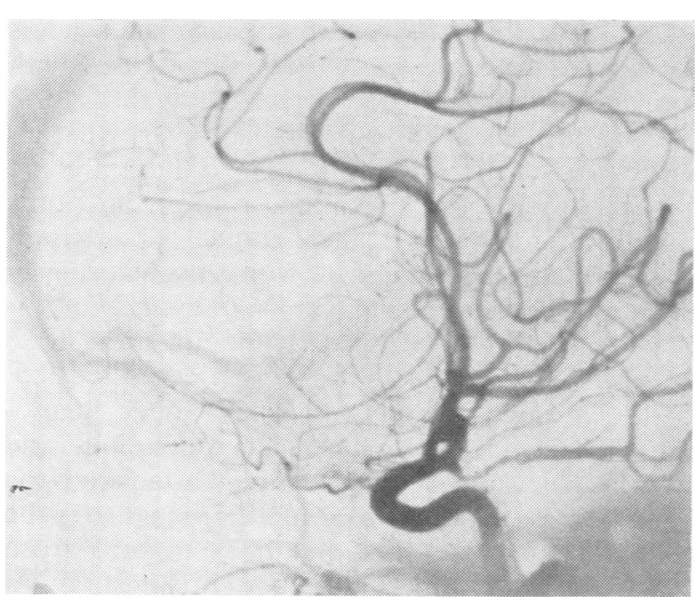

Fig. 4 Left carotid angiogram, arterial phase, lateral projection (case 7). There is a large subfrontal mass displacing the pericallosal arteries posteriorly, superiorly, and to the right, stretching apart the medial frontal branches on the inferior half of the hemisphere. There is also elevation of the first parts of both anterior cerebral arteries and lateral displacement of the intracavernous and supraclinoid segments of the internal carotid artery. Despite the fact that the latter changes indicated an intra and suprasellar mass the angiographic misdiagnosis of meningioma was made.

normally placed chiasm may not be recognised on other studies.

\section{Treatment and results}

Surgery was performed through either a frontal or frontotemporal craniotcmy using either a subfrontal or combined subfrontal-subtemporal approach and microneurosurgical technique. The primary aim was radical excision of the intradural part of the tumour to decompress the optic apparatus and hypothalamus. When this was impossible (five cases) the tumour was removed subtotally to diminish its bulk (four cases). In one case only a limited biopsy for histological purposes could be performed. Patients with gross hydrocephalus were treated with postoperative ventricular drainage aiming to convert later to a ventricular shunt if needed (case 11). Dexamethasone $4 \mathrm{mg} 6$ hourly was started before the operation and carried on throughout the postoperative period in decreasing doses. All surviving patients received $\mathrm{x}$-ray therapy.

Three patients (cases 10,12, and 15) all with massive lobulated hypothalamic and posterior extensions, died in the immediate postoperative period (operative mortality $18.7 \%$ ). Two patients died within six months of surgery; case 11, four months after a full course of deep radiotherapy, and case 3 suddenly three months after surgery, probably as a result of pulmonary embolism.

Length of follow-up ranged from 10 years down to six months (mean 3 years). Ten patients are well and leading normal lives; a further case from overseas has been lost to follow-up. No case showed deterioration of vision in our scoring system postoperatively, but with one exception (case 9), whose vision returned to normal, improvement was moderate (seven patients) or absent (five patients).

Histological examination of the tumours showed that 14 were typical chromophobe adenomas and two (cases 7 and 13) were mixed chromophobe and acidophil adencmas (Table 1).

\section{Discussion}

The accumulated experience of surgeons since Jefferson's (1940) first report has made it clear that the mortality of excision of very large pituitary tumours is significantly higher than those with modest extrasellar extensions (Bakay, 1950; Jefferson, 1969; Wirth et al., 1974). From the current series of cases, we have concluded that gross thalamic and intra-third ventricular extension with displacement of the upper brainstem, should be considered a contraindication to surgery (Fig. 5), unless access to the main tumour can be obtained through another, for example subfrontal (Fig. 6) or temporal, extension, and the consistency of the mass is such that its bulk can be radically removed. If this is not the case, simple biopsy, shunting, and radiotherapy may be the only recourse.

Partial excision is inadvisable. Blood supply to the distal part of the tumour is thereby jeopardised causing its infarction and swelling, with even more compression of the upper brainstem. All our immediate postoperative mortality was confined to such cases (cases 10, 12, and 15).

We have no experience of attempted treatment of these cases with bromocriptine, but in the presence of a gross mass lesion in the third ventricle, perhaps with associated hydrocephalus, it seems clear that at any rate the hydrocephalus must be treated. Possible benefits of the more modern medical management will have to await further trial. As Guiot has pointed out (Guiot and Derome, 1976), if such tumours with gross hypothalamic and posterior extensions are associated only with downward spread into the 


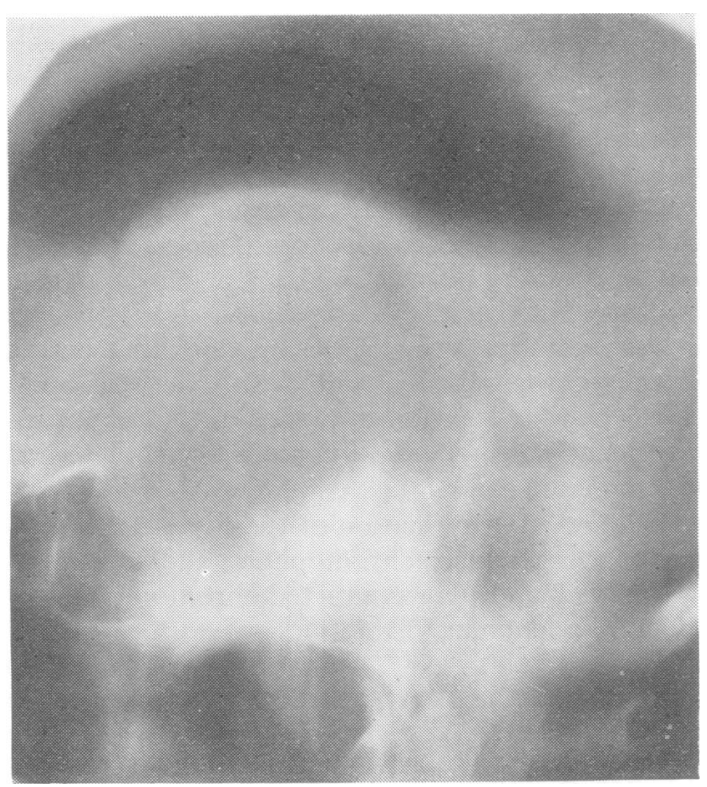

Fig. 5 Pneumoencephalogram, erect position (case 12). Lateral midline tomogram. A large tumour elevates the third ventricle and impresses into the floor. The aqueduct is displaced posteriorly.

sphenoidal sinus, a transphenoidal approach may produce adequate decompression. He has reported an occasional case supporting this conclusion. Transphenoidal surgery was not considered in any of our cases, but could scarcely have had a less satisfactory outcome than these three cases with gross brainstem distortion attacked by the suprasellar route. It is open to doubt, however, whether multiple extensions extradurally or laterally are accessible by the transphenoidal route, and it may be that a combined transphenoidal and supratentorial approach would be worth considering in selected circumstances.

The aim of radical surgery in our own series was also substantially that of visual preservation. To this end an attempt was always made to produce an adequate chiasmal decompression, and this was best achieved by a direct attack on the largest extrasellar portion of the mass. If the mass, for example, lies in the temporal fossa (Fig. 7), a direct temporal approach is best, and a small temporal lobectomy frequently facilitates approach to a giant retrochiasmatic lesion as well as to one in the temporal fossa. Otherwise, a frontal approach on the non-dominant side with the capacity to extend if necessary behind the carotid artery into the anterior temporal fossa, seems to provide the most generous access. It is particularly worth pointing out that intradural extension into the posterior fossa (Fig. 8) is often easier dealt with on the left side from a right subfrontal approach where the oblique line of sight between the nerves or lateral to the nerve on the right, carries one more readily into the left side of the tentorial hiatus than is the case

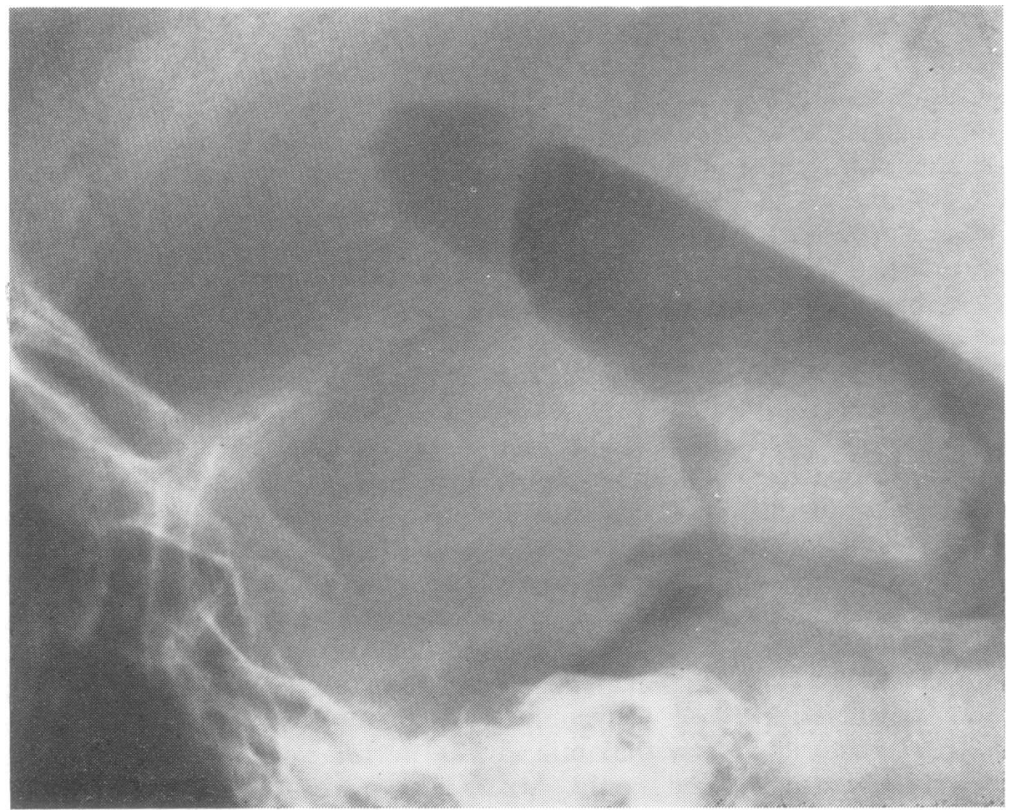

Fig. 6 Lateral brow-up ventriculogram (case 9). The anterior horns are displaced posteriorly and superiorly. The third ventricle is displaced markedly posteriorly. Access to the large posterior extension was facilitated through the continuous suprasellar and subfrontal mass. 
with a right handed surgeon operating from the left. For such a mass going posteriorly on the right side, additional access by partial temporal lobe resection may be required.

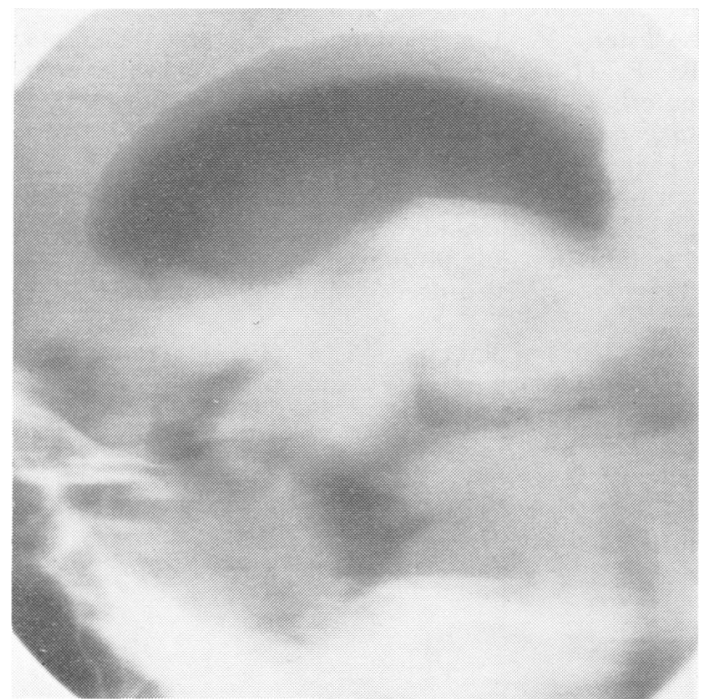

Fig. 7 Pneumoencephalogram (case 13). Brow-up, lateral tomogram through left temporal horn. The mass extending through the Sylvian fissure had displaced the temporal horn posteriorly.

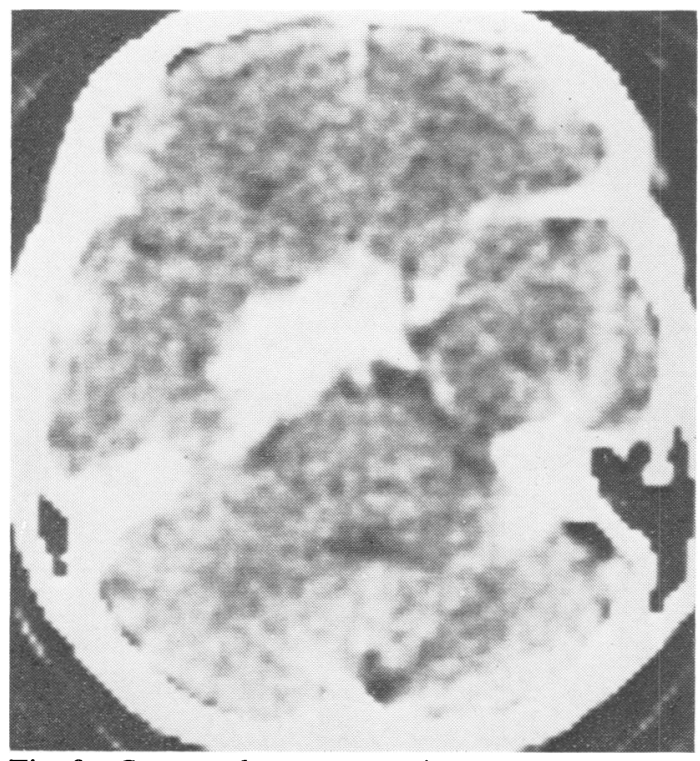

Fig. 8 Computed tomogram after intravenous contrast medium (case 16). Mass of increased attenuation in the suprasellar, left parasellar, and medial temporal regions extending into the left side of the posterior fossa.
From consideration of the tumours in our own series, the most significant points in decision for a particular surgical approach must be the detailed analysis of the exact method of spread. In this respect, although available only in the later tumours in our series, computerised tomography with contrast enhancement appears to be the most significant single investigation. It is, however, disappointing that CAT scan has not so far enabled us to predict the likely consistency of the tumour. Even very large extrasellar extensions which are soft are surprisingly amenable to radical excision. While in pituitary tumours generally, hard collagenous lesions are not common, it seems unfortunate that a relatively higher proportion of the giant extrasellar extensions are hard and intractable surgically, presumably because of greater age.

The exact shape, and in particular the exact nodularity of the upper portion of tumour extension can still be only adequately defined by detailed high-quality air study, and our recommendation would still be for pneumoencephalography. Other authors (McAllister et al., 1978) might consider that computerised metrizamide cisternography offers a logical alternative. Such air study, in addition to providing detailed anatomy of the upper margin and the extent of the lesion, will usually provide a clue to the site of the chiasm, whose relationship to such large masses may be quite difficult to predict on clinical grounds from visual field tests.

The role of angiography seems to us to lie principally in defining the detailed anatomical relationships of the sub- and intracavernous portion of the mass, the prediction of the likelihood of close investment of major cerebral vessels, and the exclusion of coincidental vascular pathology such as aneurysm.

The surgical management of the giant pituitary tumour presents technical problems entirely different in magnitude from the more usual pituitary mass. Nevertheless the 10 followed survivors of radical surgery in our series are leading a normal life up to 10 years after surgery, with some degree of visual recovery in seven, and preservation of status quo in three cases. This appears to justify consideration of direct attack with radical removal in mind, irrespective of the sheer bulk of tumour, particularly in the younger age group.

\section{References}

Bakay, L. (1950). The results of 300 pituitary adenoma operations (Professor Herbert Olivecrona's series). Journal of Neurosurgery, 7, 240-255. 
Guiot, D., and Derome, P. (1976). Surgical problems of pituitary adenomas. In Advances and Technical Standards in Neurosurgery, vol. 3, pp. 3-33. Edited by $H$. Krayenbuhl. Springer-Verlag: Wein, New York.

Jakubowski, J., and Kendall, B. (1978). Coincidental aneurysms with tumours of pituitary origin. Journal of Neurology, Neurosurgery, and Psychiatry, 41, 972-979.

Jefferson, A. (1969). Chromophobe pituitary adenomata The size of the supracellar portion in relation to the safety of operation. Journal of Neurology, Neurosurgery, and Psychiatry, 32, 632 (abstract).

Jefferson, A. (1978). Transcranial operation and radiotherapy of pituitary adenomas. In Treatment of Pituitary Adenomas: A European Workshop Edited by R. Fahlbush and K. V. Werder. Thieme Verlag: Stuttgart.

Jefferson, G. (1940). Extrasellar extensions of pituitary adenomas. President's address. Proceedings of the Royal Society of Medicine, 33, 433-458.

Jefferson, G. (1955). The invasive adenomas of the anterior pituitary. University of Liverpool. The
Sherrington Lectures III. University Press: Liverpool.

Kunicki, A., Grochowski, W., and Czernicki, Zb. (1975). Szerzenie sie gruczolakow przysadki mozgowej poza siedeko tureckie. Neurologia Neurochirurgia Polska, 9, 626-629.

McAllister, V. L., Hankinson, J., and Sengupta, R. P. (1978). Problems in diagnosis with computerised tomography (CT). In Advances in Neurosurgery, vol. 6, pp. 61-74. Edited by $R$. Wüllenweber, H. Wenker, M. Brock, and M. Klinger. Springer-Verlag: Berlin, Heidelberg, New York.

Ogilvy, K., and Jakubowski, J. (1973). Intracranial dissemination of pituitary adenomas. Journal of Neurology, Neurosurgery, and Psychiatry, 34, 199205.

Symon, L., and Jakubowski, J. (1979). Transcranial management of pituitary tumours with suprasellar cxtension. Journal of Neurology, Neurosurgery, and Psychiatry, 42, 123-133.

Wirth, P., Schwartz, H. G., and Schwestschenau, P. R. (1974). Pituitary adenomas: factors in treatment. Clinical Neurology, 21, 8-25. 